\title{
MTSS: multi-path traffic scheduling mechanism based on SDN
}

\author{
XU Xiaolong ${ }^{1, *}$, CHEN Yun ${ }^{2}$, HU Liuyun ${ }^{3}$, and KUMAR Anup ${ }^{4}$ \\ 1. Jiangsu Key Laboratory of Big Data Security \& Intelligent Processing, Nanjing University of Posts and Telecommunications, \\ Nanjing 210023, China; 2. Institute of Big Data Research at Yancheng, Nanjing University of Posts and Telecommunications, \\ Yancheng 224000, China; 3. School of Computer Science, Nanjing University of Posts and Telecommunications, \\ Nanjing 210023, China; 4. Department of Computer Engineering and Computer Science, University of Louisville, \\ Louisville KY40292, USA
}

\begin{abstract}
Large-scale and diverse businesses based on the cloud computing platform bring the heavy network traffic to cloud data centers. However, the unbalanced workload of cloud data center network easily leads to the network congestion, the low resource utilization rate, the long delay, the low reliability, and the low throughput. In order to improve the utilization efficiency and the quality of services (QoS) of cloud system, especially to solve the problem of network congestion, we propose MTSS, a multi-path traffic scheduling mechanism based on software defined networking (SDN). MTSS utilizes the data flow scheduling flexibility of SDN and the multi-path feature of the fat-tree structure to improve the traffic balance of the cloud data center network. A heuristic traffic balancing algorithm is presented for MTSS, which periodically monitors the network link and dynamically adjusts the traffic on the heavy link to achieve programmable data forwarding and load balancing. The experimental results show that MTSS outperforms equal-cost multi-path protocol (ECMP), by effectively reducing the packet loss rate and delay. In addition, MTSS improves the utilization efficiency, the reliability and the throughput rate of the cloud data center network.
\end{abstract}

Keywords: cloud data center, software defined networking (SDN), load balancing, multi-path transmission, OpenFlow.

DOI: $10.21629 / J S E E .2019 .05 .14$

\section{Introduction}

In recent years, the growing popularity of mobile Internet, Internet of Things, social networks, big data and smart city applications, relies on the cloud computing platforms [1-3] with large-scale computing and storage capacities. Amazon, Google and Microsoft, etc., have built cloud data centers with millions of servers.

A cloud data center usually contains lots of server clus-

\footnotetext{
Manuscript received July 17, 2018.

*Corresponding author.

This work was supported by the National Key Research and Development Program of China (2018YFB1003702), the National Natural Science Foundation of China (61472192), and the Scientific and Technological Support Project (Society) of Jiangsu Province (BE2016776).
}

ters, and each of them includes thousands of servers, which are connected together via switches and routers. The cloud computing system achieves the integration of computing, storage, information resources, which makes the cloud data center a concentrated place for data communications. There is a lot of incoming and outgoing traffic between servers in the cloud data center, bringing huge workload to switches and routers, which affects the quality of services of a cloud computing system. At present, the network traffic is not balanced in the cloud data center as a result on one hand, there are many redundant idle links, but on the other hand, some links are often congested.

In the cloud data center, a variety of network protocols and a large number of network devices make the communication infrastructure more complex. This makes it difficult to configure and manage hundreds of network devices efficiently. In addition, the current network routing proto$\mathrm{col}$, such as the open shortest path first (OSPF) protocol [4], cannot effectively use potential redundant paths to improve the network throughput or reduce the communication delay [5]. The multi-path protocol, such as the equalcost multi-path (ECMP) protocol [6], can utilize the idle path, but cannot allocate the traffic from the perspective of the global network, leading to the unbalanced network traffic, and the network congestion [7].

Software defined networking (SDN) [8,9] has become a popular paradigm for centralized control in many modern networking scenarios such as cloud data centers. SDN separates the control function from the data function of network devices, and can flexibly set the network traffic path from the code level. Compared with the distributed flow control, the SDN controller has the global network information and can control the network traffic more accurately, dynamically, flexibly and automatically.

In this paper, in order to improve the utilization efficiency and the quality of services (QoS) of the cloud sys- 
tem, especially to solve the problem of network congestion, we propose MTSS, a multi-path traffic scheduling mechanism based on SDN. MTSS utilizes the data flow scheduling flexibility of SDN and the multi-path feature of the fat-tree structure [10] to improve the traffic balance and the utilization rate of the cloud data center network. A heuristic traffic balancing algorithm is presented for MTSS, which periodically monitors the network link quality and dynamically adjusts the traffic on the heavy link to achieve adaptive data forwarding and load balancing.

The rest of the paper is organized as follows. Section 2 introduces the network topology of the cloud data center, as well as the related research about multi-path routing algorithms. Section 3 describes MTSS, the multi-path traffic scheduling mechanism based on SDN proposed in this paper. In Section 4, experimental results and performance analysis are presented. Finally, Section 5 concludes the paper by summarizing the main contributions of this paper and giving the future direction of this work.

\section{Related work}

\subsection{Non-heuristic load balancing algorithms}

The current load balancing technologies usually disperse traffic based on the multi-path feature of the network [11-13]. The load balancing algorithms include the nonheuristic load balancing algorithms, heuristic load balancing algorithms and SDN-based load balancing algorithms.

Chiesa et al. [7] proposed a data transmission mechanism based on ECMP, which identifies all available paths, and selects one path according to the result of the hash operation of the specific field of the packet header. All packets of the same stream use that path, so that the order of data packets to the destination host is guaranteed. ECMP makes full use of the diversity of the transmission path in the network and distributes the data stream on the equivalent paths, to avoid wasting the bandwidth of the network. When the network is dominated by small and medium dataflows, ECMP can also show good performance. However, ECMP assumes that each path has the same transmission capacity, without considering the link bandwidth utilization, which results in the collision when two or more streams are output from the same port. This defect leads to the fact that the network is still prone to congestion even if the average link utilization is relatively low. Greenberg et al. [14] proposed the valiant load balancing (VLB) algorithm, which is a static random network load balancing algorithm. Similar to ECMP, VLB lacks the control of the global network, so there are similar problems. The performances of both ECMP and VLB depend essentially on the size and the number of data flows. Therefore, it is difficult to deal with massive data transmission due to unbalanced load and network congestion. It is imperative that more appropriate data flow scheduling strategies are needed to avoid the network bottleneck [15].

\subsection{Heuristic load balancing algorithms}

Al-Fares et al. [15] proposed Hedera, a scalable, dynamic flow scheduling system, which uses a multi-path scheme based on the simulated annealing algorithm to perform a probabilistic search to efficiently compute paths for various flows. Resource competition and conflicts in datacenter networks are frequent and intense, and become inevitable when mixing elephant and mice flows on shared transmission paths, resulting in arbitration between throughput, latency and performance degradation [16]. However, Hedera only considers elephant flows, converges slowly sometimes and cannot adapt to the network with the requirement of rapid response.

Lin et al. [17] proposed a new multi-path routing algorithm based on the ant colony optimization (ACO) algorithm improved with the traffic engineering. In this approach, as a next hop is selected, the utilization ratio of the router's buffer queue is added into the selection criterion; the utilization ratio of link is introduced to update the global pheromone; multi-paths are selected to transfer data. However, if the network load is not heavy, its performance will decline and be worse than some traditional routing algorithms.

Overall heuristic based load balancing algorithms have high complexity and slow convergence rate.

\subsection{SDN-based load balancing algorithms}

With the development and popularization of SDN and OpenFlow [18] technology, it is possible to apply SDN to the network load balancing. Domestic and foreign scholars have further studied the data flow scheduling, and network load balancing in the OpenFlow network environment.

Chen et al. [19] proposed a multi-path transmission mechanism based on OpenFlow by using OpenFlow to control the whole network, which optimizes the resource allocation from the global network perspective, and calculates the transmission path for each flow. According to the CPU utilization rate of the controller, the cycle of global load balancing is developed, which can effectively balance the network management granularity and the load of processing tasks. However, the algorithm attempts to find all reachable paths of one flow, in order to find the lightest load path. This algorithm is not only time-consuming, but also heavily dependent on the global network topology. Once the topology changes, it is necessary to recalculate all reachable paths between each pair of hosts, and the al- 
gorithm does not even mention how to find the reachable path.

Subramanian et al. [20] compared three different scheduling algorithms: random scheduling (RS), round robin ( $R R$ ), weighted round robin (WRR), in order to solve the load imbalance. The three algorithms are applied to the optimal scheduling of flow in the SDN environment, and the results show that WRR is the best.

Li et al. [21] presented a dynamic load balancing (DLB) algorithm, to efficiently schedule flows. DLB utilizes the hierarchical feature of fat-tree networks to recursively search for a path, and makes the decisions based on realtime traffic statistics obtained via the OpenFlow protocol. This dynamic strategy can bring better effect to the network load balancing than the static algorithm. However, DLB uses a local optimal strategy, and the greedy algorithm is used to select the most idle link. This approach lacks the global view, and can easily fall into local limitations, which may lead to some heavily loaded links. This could result in network congestion.

Long et al. [22] proposed the method of judging network congestion and the dynamic path optimization algorithm under the SDN framework. The threshold calculation is established as a basis for judging whether the network load is balanced. Based on this calculation, a data flow on the congested link is rerouted. The variance of the whole network link load is used as a criterion to judge whether the network is congested. This process of computation of network congestion makes the algorithm more complex, less flexible and less accurate.

\section{MTSS}

\subsection{Fat-tree}

In the cloud data center, the traditional tree structure of the network easily leads to frequent congestion, high packet loss rate, and long transmission delay [19]. In order to solve these problems, we design the new network topology based on the fat-tree structure [22]. The fat-tree-based network has a multi-level hierarchical structure, so that the network has a certain degree of fault tolerance. It can provide a number of paths for hosts, which can reduce the load on each link to prevent the network congestions.

Fat-tree includes three layers [23]: the core layer, the convergence layer, and the edge layer. As shown in Fig. 1, there are four performance optimization datacenters (POD). Each POD is a complete bipartite graph, consisting of convergent switches and edge switches. The topology of fat-tree brings network the diversity of available paths, which means there are many equivalent paths be- tween each pair of hosts.

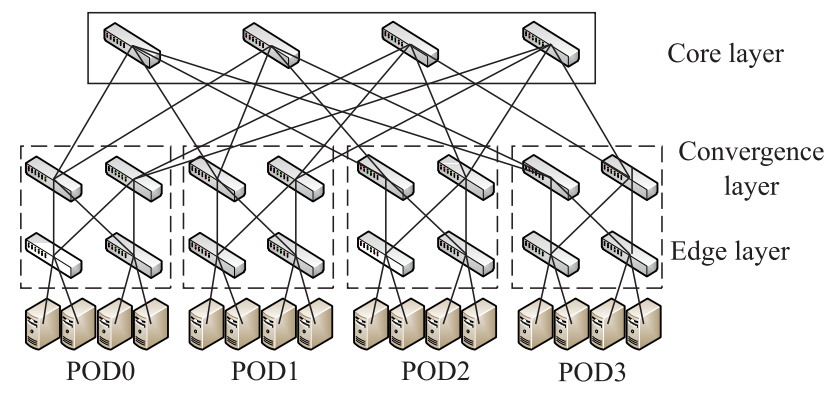

Fig. 1 Topology of fat-tree

\subsection{SDN}

The SDN $[8,9,24]$ is different from the traditional network schemes. In the traditional network, each router has both the control plane and the data plane. These routers exchange routing entries with each other based on the routing protocol. The control plane is distributed on each network device, so that the global network cannot be controlled effectively. SDN uses the OpenFlow protocol [18] to separate the control plane from the data plane. Switches are only responsible for the data plane, which receives flow tables sent by the controller, and forwards data according to the flow entries. Because switches do not need to understand every kind of protocols or how to make the flow tables, their workload is reduced and their efficiency is improved. The controller provides a flexible programmable interface, which can be used to update the flow tables, so that users can develop suitable data forwarding strategies. This allows improved network management and control capabilities.

The control function of an SDN-based network is concentrated in the controller, which knows the topology and state of the whole network system. The performance of the controller directly affects the operating efficiency of the entire data center. As SDN gets more attention, many new types of SDN controllers are being developed. Typical SDN controllers include Floodlight [25], OpenDaylight [26] and POX [27], etc.

\subsection{Model and algorithm}

The traffic scheduling algorithm based on SDN is presented here in detail. Floodlight is used in MTSS to send and update the flow tables of Open vSwitch (OVS) switches, and control the forwarding of data flows. If a new data flow joins the system, MTSS will get the traffic of each link in the network real-time, search out the best path, and then distribute flow tables. At the same time, MTSS periodically monitors the traffic of each link. If a link is found with heavy load, MTSS will modify flow tables in 
all switches on the path, and then transfer some data flows to another path, so as to achieve load balance and improve the network throughput.

The model of MTSS is shown in Fig. 2. The main modules of MTSS are deployed on the Floodlight controller, including the load monitoring module, the path optimization module and the flow table module, which cooperate with each other to provide relevant information for the other modules. The load monitoring model monitors the load of the link in real time and periodically queries and collects network information of the switches in the network topology to form the load matrix. The source address of a data stream with a large flow rate in a link with a load exceeding the threshold is extracted and then sent to the path optimization module with the load matrix. The path optimization module receives the whole link load matrix and source address after the load monitoring module is processed, and then calculates the best path between source and host based on current link load. After the path optimization module calculates the optimal path, the flow table hair module is responsible for sending the flow table to the switches on all paths.

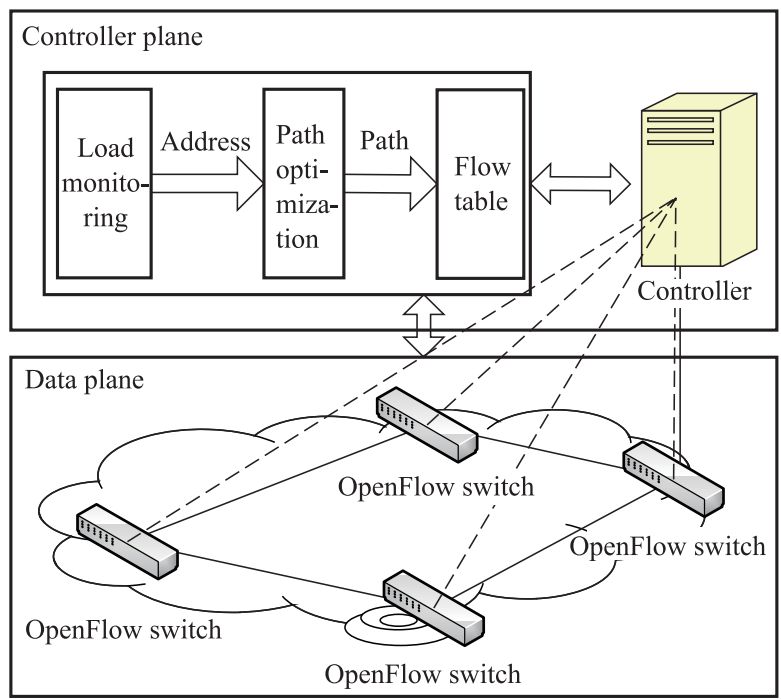

Fig. 2 Model of MTSS

(i) Load monitoring module

The monitoring module is responsible for periodically collecting the network information of switches using the statistical function of OpenFlow. The module can conveniently obtain all kinds of network information. As shown in Fig. 3, an OpenFlow switch is mainly composed of the security channel, the flow table and the OpenFlow protocol. The counter of the flow table is used to count the number of processed packets. There are four kinds of counters: the counter for flows, the counter for switch ports, the counter for flow entries and the counter for queues. The number of data packets and the throughput of switch can be obtained using these counters.

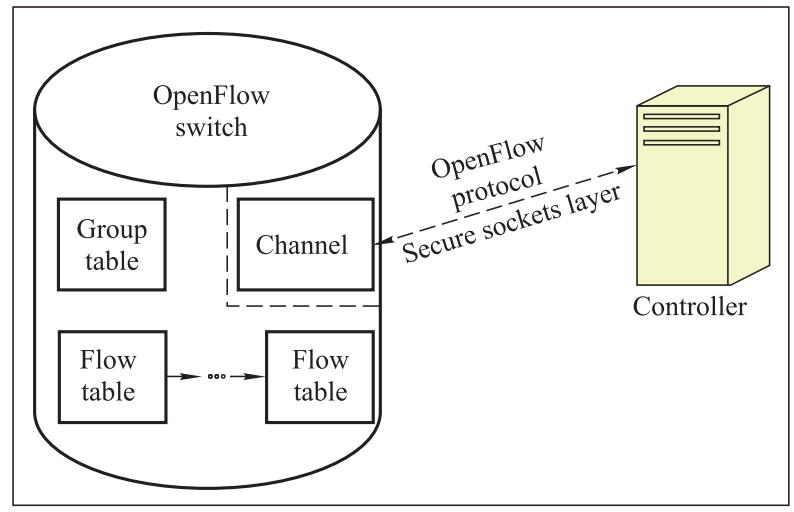

Fig. 3 Structure of OpenFlow switch

This module computes the throughput of each port and the load of each link between switches, and stores these data items in the load matrix. If the utilization rate of a link exceeds the threshold, the module will extract the source and destination address from the flow table entries, so that the path optimization module can evaluate the optimal path.

The threshold of link utilization can be taken as the degree of tolerance. If the threshold is set too high, the link may easily be congested, and multiple paths of the fat-tree network are not fully utilized. On the other hand, if the threshold is set too low, the paths of data flows may change too frequently [28].

(ii) Path optimization module

The path optimization module receives the full link load matrix and the source and destination addresses from the load monitoring module, then calls the algorithm proposed in this paper to calculate the optimal path between the source and destination hosts based on the current link load.

The scheduling approach is a heuristic algorithm based on population. For $N$ particles in the population, the effect of a single particle is limited, but the interaction between the particles makes the overall search diversification, which greatly improves the performance. Each particle is an abstract concept that represents a path, from the source address to the destination address. Each path consists of a number of nodes, and each node is switch in the network. We use $X_{j}(j=1,2, \ldots)$ to represent a node. In the process of searching, the particles tend to move closer to their optimal solution and the global optimal solution, and adjust the next node in the path according to their own historical optimal solution and the global optimal solution of the whole population. Finally, the optimal solution of all particles is the optimal path.

Supposing there are $N$ particles in the population, for the $i$ th particle, denoted by $\left[X_{1}, X_{2}, \ldots, X_{j}, \ldots, X_{n}\right]$, 
$X_{j}$ represents a switch on the path, and all the adjacent switches of $X_{j}$ are candidates of the next node $X_{j+1}$. The candidate nodes have different probabilities. The particle will choose one with a certain probability rule, whose switch corresponding to the higher probability will be more likely to be selected as the next node.

After several iterations, the algorithm will find the optimal solution. If the iteration phase is different, and the probability of each candidate node will be calculated in different ways. When the iteration time $T$ is equal to 0 , assuming the current switch on the path that the $i$ th particle searches is $X_{j}$, the probability of $X_{j}$ 's candidate node will be calculated according to the link load between $X_{j}$ and its adjacent switches. Then we generate a random number, determine the next switch of $X_{j}$, repeat the above process, and continue selecting the next switch for the current switch until finding the destination node. Then, we continue searching a path for $N$ particles, and each path is the local optimal path of each particle. In the evaluation function as shown in (1), we calculate the evaluation value of each particle, and then select the path with the maximum value from the $N$ paths as a global optimal path.

$$
\left\{\begin{array}{l}
F=\frac{S}{L} \cdot \frac{D-\omega^{*}\left(L-L_{\min }\right)}{D} \\
D=L_{\max }-L_{\min }
\end{array}\right.
$$

where $S$ represents the total available bandwidth of the path, and $L$ represents the number of links contained in the path. A problem is easy to appear in the search path, that is, the particles excessively pursue available bandwidths, which causes that the path may be long, and the times of forwarding may be too much. It will affect the data transmission efficiency, and increase transmission delay. In this paper, we set the adjusted coefficient to balance the relationship between available bandwidth and path length, in which, $L_{\max }$ represents the maximum length of all reachable paths, $L_{\min }$ represents the minimum length of all reachable paths, and $D$ represents the difference between the maximum and the minimum length. $\omega(0 \leqslant \omega \leqslant 1)$ is the key factor of the adjusted coefficient, which adjusts the influence of the path length on the adjusted coefficient, thereby affecting the final evaluation value. This can control the bias of the algorithm, that is, the preference for available bandwidth, or the shortest path. If $\omega$ is smaller, the influence of the path length factor is weak in the algorithm until the minimum value, then the optimal path algorithm no longer considers the influence of the path length on the selected path. The bigger $\omega$ is, the greater the impact on the evaluation value is, making the path length tend to $L_{\text {min }}$. To the actual network situation, the value of $\omega$ is crucial to the performance of the algorithm.
When the iteration time $T$ is greater than 0 , the method of probability calculation is shown in (2). A new probability is calculated by combining the probability of the previous iteration and whether the node appears in the local optimal path or the global optimal path. According to the same steps as the 0th iteration, each switch in the corresponding path of $N$ particles is selected. Then, according to (1), the new evaluation value of each particle is calculated. Finally, according to the evaluation value, we update the local optimal path of each particle and the global optimal path of the whole population. Then, the above same steps are performed for each iteration.

$$
\left\{\begin{array}{l}
p_{x}=\left[p_{y}+\left(n_{1} \alpha+n_{2} \beta\right) /\left(n_{3} \alpha+n_{4} \beta\right)\right] / 2 \\
n_{1}, n_{2}=1 \text { or } 0
\end{array}\right.
$$

where $\alpha$ is the weight of the node which appears in the global optimal path, and $\beta$ is the weight of the node appearing in the local optimal path. $n_{1}$ and $n_{2}$ respectively are the number of nodes appearing in the global and local paths. $n_{3}$ and $n_{4}$ respectively represent the total number of adjacent nodes appearing in the global and local paths. $p_{y}$ is the probability of the last iteration of each particle, $p_{x}$ is the new probability of this iteration. Equation (2) introduces $\alpha$ and $\beta$ to avoid the shortcoming that the algorithm is easy to trap in local optimum, which leads to the lack of global search ability. $\alpha$ guides the particle to move closer to the global optimal path, which avoids being trapped in the local optimum, $\beta$ guides the particle to explore along its own optimal direction, which expands the path diversity and avoids the particle ending the search early.

The specific steps of path optimization are as follows:

Step 1 First, the source and destination switches are determined, and the $N$ particles are set, and then the times of iterations $T$ is initialized to 0 .

Step 2 Query the entire topology, and initialize the adjacent nodes table.

Step 3 The available bandwidth of each link is obtained by the load monitoring module, and the available bandwidth table is established.

Step 4 For each particle, the source switch is set to be the first node in the path, in the search process, the last node of the path that has been searched is called the current node.

Step 5 When $T=0$, according to the available bandwidth table and adjacent node table, the probability of the adjacent node of the current node is calculated. Otherwise, the probability of the adjacent node is calculated according to $(2)$.

Step 6 The random number $X$ is generated, and the next node is determined according to the probability of each adjacent node of the current node. In order to prevent the particles from falling into the dead cycle while 
finding the path, a node that has already appeared on the path is put into a forbidden table, and the subsequent node will not select the node which is in the forbidden table. If a node in the table is selected, Step 6 will be repeated until the selected node is not in the forbidden table. Of course, there may be such a situation, in the search process, the adjacent nodes of the current node are in the forbidden table, the particle will have no way to go, at this time the road is invalid, jump directly to Step 7 .

Step 7 The selected node is put into the path, jump back to Step 5, until the selected node is the target node.

Step 8 If the path is invalid, the evaluation value is 0 , according to (1), the evaluation value of this particle is calculated, then, jump back to Step 4, the corresponding path of $N$ particles is searched and the evaluation value is calculated. When $T=0$, the corresponding path of each particle is the local optimal path, the best path of $N$ paths is the global optimal path. Otherwise, the evaluation value of each particle corresponding path in the $T$ th iteration and its local optimal path are compared, and the evaluation value of all paths in the $T$ th iteration and the global optimal path are compared with the $T$ iteration, then update the local optimal path of each particle and the global path of the $T$ th iteration.

Step 9 If $T$ is equal to the preset number of iterations, skip to Step 10, otherwise $T$ plus 1, jump back to Step 4, update the local optimal path of each particle and the global path in each iteration.

Step 10 When the iteration is over, the whole process is over, the current global optimal path is the optimal path searched by the algorithm in this paper.

According to (1), the evaluation value of each particle is calculated. Then, the path with the largest value of evaluation will be selected as the global optimal path from the initial $N$ path. The global optimum is from the local optimums in the later iteration searches. Equation (2) utilizes two coefficients, $\alpha$ and $\beta$. In the process of iteration, the particle is guided to the global optimal path by $\alpha$, while the particle is guided to the local optimal path by $\beta$. After continuous iterations, the result gets closer to the optimal path. Although the solutions are not always the best solution, they are approaching the optimal solution.

To facilitate a better understanding of the algorithm, we provide its pseudocode as follows:

Algorithm: Path optimization algorithm

Input: SrcSwitch, DesSwitch, $n$, bandWidth

Output: Optimal path

1: $N=$ get Number (SrcSwitch, DesSwitch)

2: $i=0$

3: Initialization $(N)$

4: Path = []
5: While $(i<n)$

6: $\quad$ For $j$ in $N$ do

7: $\quad$ j.setFirst Node (SrcSwitch)

8: $\quad$ proList $=[]$

9: $\quad$ While $($ nextNode $!=$ DesSwitch $)$

10: $\quad$ If $(i==0)$

11:

12:

13:

14:

15:

16:

17:

18:

19:

20:

21:

22:

23:

$p=$ getProbability (bandWidth)

Else

$p=$ getProbability 2()

End

nextNode $=$ Random $(p)$

If (nextNode in proList)

nextNode $=$ Random $(p)$

Else

j.append(nextNode)

End

End

Path. append $(j)$

24: OptPath = getMaxEvaluation $($ Path $)$

25: $i++$

26: End

27: return OptPath

In the pseudocode of the path optimization algorithm, get Number () returns the number of paths between source switch and destination switch; Initialization() initializes all particles, and each particle represents a link; setFirstNode() sets the starting for each link; getProbability () returns the probability of the adjacent node of the current node; getProbability2() calculates the results with (1); getMaxEvaluation() returns the optimal path by calculating evaluation values of all paths.

(iii) Flow table module

After the optimal path is searched by the path optimization module, the flow table module is responsible for sending the flow table to the switches on the path. OpenFlow supports two ways to insert the flow table: active and passive, when the packet arrives at the OVS switch, if it does not match to any flow table entry, the controller will passively insert the flow table. In this paper, we use the active way to insert the flow table, that is, the flow table will be send before the packet arrives at the switch. Static Flow Pusher is used as the function module of the Floodlight controller, which usually adopts the active way. The module exposes the REST application programming interface (API) to the user, the user can call API to interact with the controller, and control the data forwarding direction. This will be able to migrate the data flow on the heavier load path to the lighter load path, thus to achieve the purpose of network load balancing. 
In the process of updating the flow table, the path must ensure the seamless handover, that is, there is always a connected path. We should avoid the data flow in the table that does not match any flow table as this will affect the transmission delay and cause packet loss. Therefore, we need to adopt a certain way to avoid this problem in the process of updating the flow table. Here are two ways to meet this requirement:

The first option is to set the priority of the new table lower than that of the old flow table. We sequentially delete the old flow table, and the data flow is transferred gradually to a new path in the process of adjustment.

The second option is the reverse insertion of new tables. We insert the flow table in the reverse order from the destination switch to the source switch. In the process of inserting the new table, data flow is still transmitted according to the old flow table. After inserting, the old flow table will be removed. When the old flow table is removed, the data flow will be completely transferred to the new path. Priority of the flow table ranges in $[0,65536]$. With the first method, a new priority lists below the old flow table, and 0 is the lowest priority. Long time operation may bring the new table negative values or make it unable to set priorities. Therefore, the first method is not suitable for long-term operation of the system.

The specific steps of updating flow table using the second option are as follows:

Step 1 The optimal path is obtained by the path optimization module, and all switches on the path are extracted.

Step 2 Call API to obtain network topology information, and record the connection port between switches, and between the hosts and switches.

Step 3 Set flow table entries. Each entry consists of switch media access control (MAC) address, flow name, port and other information.

Step 4 The controller will gradually send the flow table to the corresponding switch in the reverse order.

Step 5 The old flow tables are removed to avoid the flow table redundancy.

Step 6 The new forwarding rules on the switch are rapidly generated, and some of the data flows on the heavier link are converted to a lighter load.

\section{Experiments}

\subsection{Experimental environment}

In this paper, we use the network simulator and SDN controller. The Mininet2.0 [29] is selected as the network simulator, as it is a lightweight, powerful software defined network platform. It can customize a variety of network topology based on Python language, and creates SDN network with hundreds of nodes on a single computer. It is very convenient to simulate the OVS switch, and it also supports OpenFlow and OpenVswitch [30] protocol. The most important is that the Mininet platform has a high hardware portability, and provides seamless migration to the real hardware environment without making any changes. We have used Floodlight1.0 as the SDN controller. Compared to OpenDaylight, POX and other controllers, Floodlight operation is very simple and convenient.

In this simulation, Mininet constructs a fat-tree topology network, as far as possible to restore the flow of the data center. At present, there are two kinds of flow patterns, which are the stochastic model and the probabilistic model. In this paper, the stochastic model is used to simulate the network traffic, that is, the host sends packets to any host in the network with equal probability. In this paper, the network has a total of 20 switches and 16 hosts, all hosts and switches in the network, the links between the switches are full duplex links, and the bandwidth of each link is set as $100 \mathrm{Mbits} / \mathrm{s}$.

\subsection{Experimental verification}

In order to determine the appropriate $\omega$ value, we have carried out several experiments to calculate the average value, and compare the advantages and disadvantages of the algorithm under different $\omega$ values. Different $\omega$ values evaluate different optimal paths and path lengths. The experimental results are shown in Fig. 4. The time delay of data flow transmission along different paths is different. It can be clearly seen from the figure that, when $\omega$ is about 0.6 , the communication delay is the smallest. Therefore, the $\omega$ value of (1) is set to 0.6 .

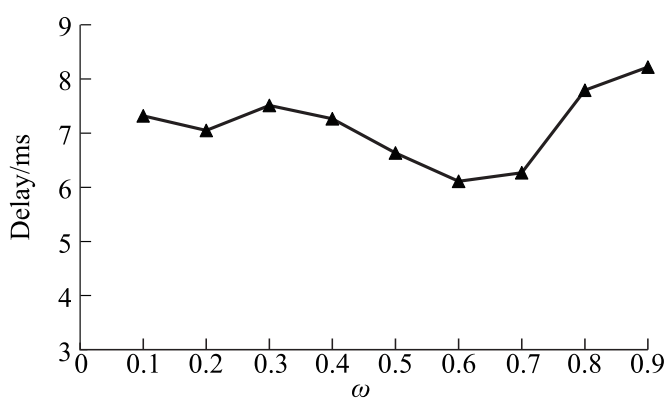

Fig. 4 Transmission delay under different $\omega$ values

In order to determine the size of $\alpha$ and $\beta$, we also carry out many experiments to get the average results, different $\alpha$ and $\beta$ values make the path and length different. We determine the ratio of $\alpha$ to $\beta$ from two aspects: i) the probability of finding the optimal path, and ii) the average number of iterations.

As shown in Fig. 5(a), three rounds of experiments are 
carried out, each experiment is performed 100 times repeatedly, and the probability of the optimal path searched by the algorithm under different ratios of $\alpha$ to $\beta$ is recorded. Finally, the average value of the three experiments is calculated. If the ratio is 1.5 , the probability of the optimal path chosen by the algorithm is the highest. The experimental results show that the ratio of $\alpha$ to $\beta$ can adjust the search ability of the algorithm, and can also test whether the particles in the algorithm can search the perimeter path adequately.

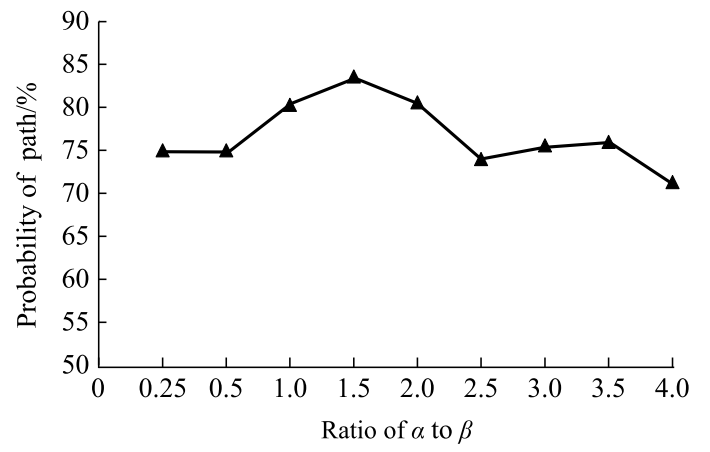

(a) Influence of the ratio of $\alpha$ to $\beta$ on probability of path

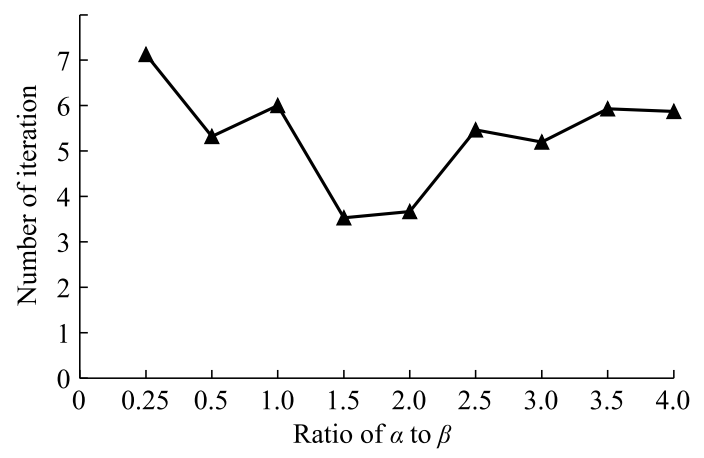

(b) Influence of the ratio of $\alpha$ to $\beta$ on the number of iterations

Fig. 5 Performance of the algorithm under different ratios of $\alpha$ to $\beta$

As shown in Fig. 5(b), we record the number of iterations that the algorithm searches for the optimal path under different ratios, when the ratio is 1.5 or 2 , the number of iterations is the least, and the optimal path could be searched quickly. The difference between the most number of iterations and the minimum iteration is not large. This is because the network size is small compared to the data center. The difference of the number of iterations will be quite large in the large data center network. In addition, during the experiment, we find that when the ratio of $\alpha$ to $\beta$ is large, the algorithm quickly enters the state of convergence after two or three iterations, and most particles are biased in favor of the same path. When the ratio is relatively small, the particle can search a lot of paths, which highlights the diversity of particles. However, the particles often search the path according to their own experience, as it lacks global view, leading to the slow convergence of the algorithm. This in turn affects the efficiency of the algorithm. Therefore, the ratio of $\alpha$ to $\beta$ in (2) is set to 1.5 .

In the network that has been built successfully, we customize the Mininet command "iperfmulti", which in turn makes each host select one host randomly, and send packets, the interval of each flow is $5 \mathrm{~s}$, the duration is $50 \mathrm{~s}$. The rate of flow increases from $10 \mathrm{Mbps}$ to $70 \mathrm{Mbps}$ with the iterative step size of $10 \mathrm{Mbps}$, and we evaluate the performance of MTSS, ECMP and DLB by comparing the network's load balancing, packet loss rate, and transmission delay and network utilization.

(i) Load balancing

The performance of network equilibrium is determined by the standard deviation of each link load of the network. If the standard deviation of the load is large, the load of the network is more imbalanced, otherwise, the load on each chain is similar, and the balance is better.

$$
\sigma=\sqrt{\frac{1}{N} \sum_{i=1}^{N}\left(U_{i}-\bar{U}\right)^{2}}
$$

where $N$ is the total number of links, $U_{i}$ on behalf of the number of $I$ has been used in the bandwidth, and $\bar{U}$ represents the average used bandwidth.

Fig. 6 shows the network load balancing performance. It can be seen from the figure that the standard deviation of MTSS is the smallest, and MTSS is always below the ECMP and DLB load under different flow rates. This shows that the flow distribution is relatively uniform and the network load is relatively balanced.

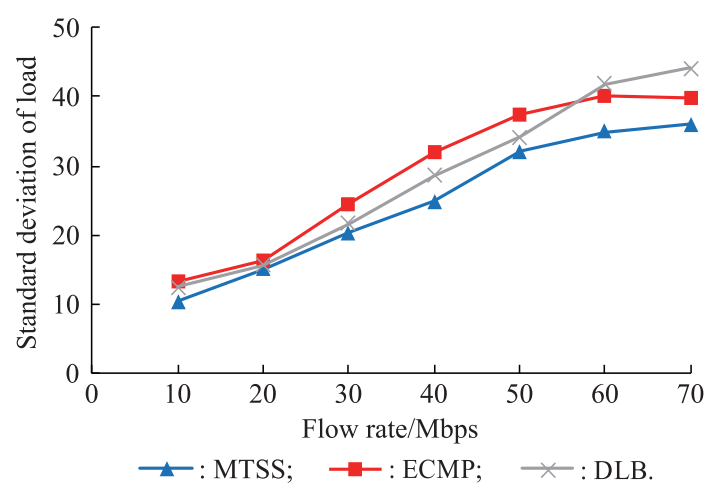

Fig. 6 Performance of load balancing

(ii) Network utilization

The network utilization is the ratio between the actual bandwidth and the specified bandwidth. The specified bandwidth is the bandwidth sent by the client, and the actual bandwidth is the bandwidth received by the server. This experiment records the average value of each flow utilization. 
Fig. 7 shows the average bandwidth utilization under different flow rates. In the three algorithms, the average utilization rates all decrease with the increase of the flow rate. When the flow rate is low, the network only has a small amount of data, and the link bandwidth is enough, so the utilizations of the three algorithms are relatively high. While the flow rate is high, the utilizations of the three algorithms all decline. However, the overall utilization rate of MTSS is higher than that of the other two algorithms. ECMP and DLB are more likely to cause network congestion, so the average bandwidth utilization is lower.

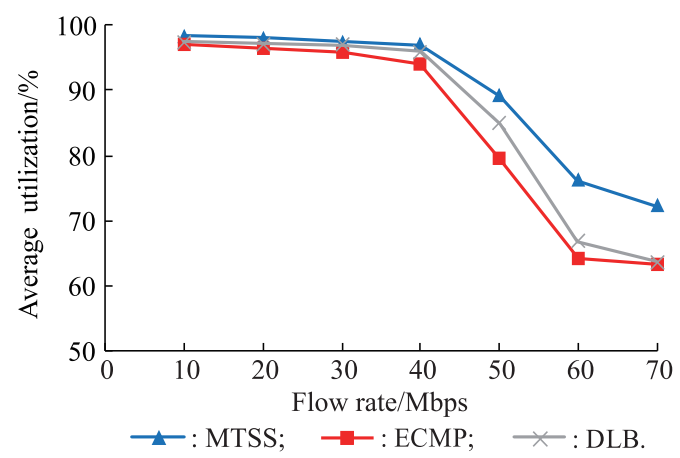

Fig. 7 Average utilization of network

(iii) Packet loss rate

The experimental results of the three algorithms in the packet loss rate are shown in Fig. 8. As can be clearly seen from Fig. 8, when the data source sending rate is lower than $40 \mathrm{Mbps}$, the packet loss rate of the three algorithms is very small, almost equal to 0 .

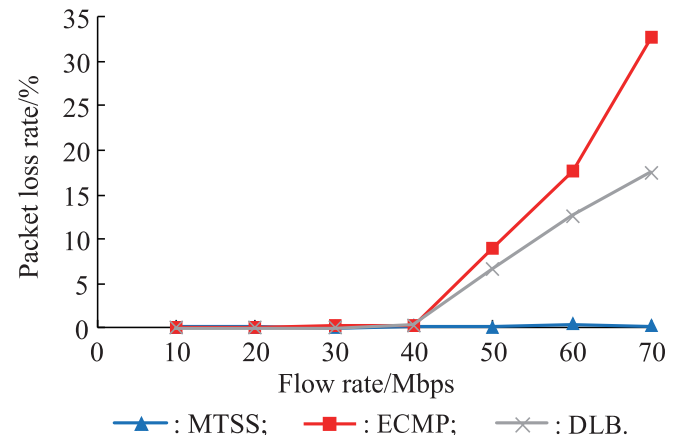

Fig. 8 Packet loss rate

However, when the data rate is greater than $40 \mathrm{Mbps}$, the packet loss rates in ECMP and DLB algorithms increase sharply, and with the increase of the flow rate, the packet loss rate is bigger and bigger. In contrast, the algorithm proposed in this paper makes the packet loss rate stable in the process of the change of the flow rate. The reason lies in the nature of their own. ECMP is prone to cause large flow collision, and DLB adopts the greedy strategy, which easily causes the available bandwidth on one link to be very small. (iv) Transmission delay

We use the Ping command to get the delay under different link loads. The experimental results are shown in Fig. 9. Different algorithms make the traffic distribution in the link different, which leads to the delay difference. As can be seen from Fig. 9, when the transmission rate is lower than $30 \mathrm{Mbps}$, the three algorithms behave the same, which shows that the lower transmission rate is, the lighter the network load is, and there is no congestion. However, with the increase of the rate, the delay of ECMP increases gradually, which indicates that the congestion is serious and the network performance is declining. Because ECMP itself determines it cannot distinguish which link is with light load, which link load is heavy, the data cannot be transfered to the lower load link. This makes the number of packets waiting in the queue increase, and results in a sharp increase in delay. However, in the case of different traffic rates, MTSS is more stable in delay, because the algorithm can get the load of each link in the whole network topology, and then switch the packet to the optimal one.

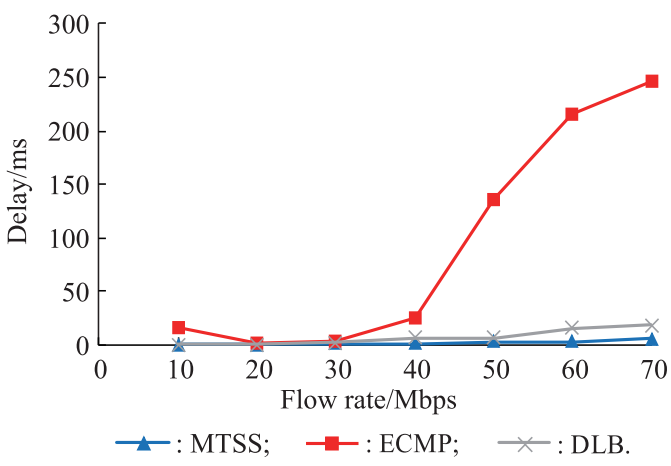

Fig. 9 Transmission delay

\section{Conclusions}

We introduce SDN to data center network management, combine the characteristics of SDN, and propose MTSS, which periodically obtains network load conditions of each link. If the link load exceeds the threshold, we will make the optimal path for some flow in the heavy link, update the flow table on the corresponding switch, and schedule part traffic to lighter load link, thereby it reduces the link load, and makes full use of the advantages of fat-tree multi-path. Through the experimental evaluation, compared with the widely used ECMP routing algorithm, the proposed MTSS algorithm can significantly reduce the network packet loss rate and transmission delay, balance the link load, and improve the network utilization.

We will further optimize the algorithm. For example, the standard deviation can be calculated as a criterion to trigger the algorithm, to overcome the defects of the constant 
threshold in this paper, and to achieve better results under different load conditions. In addition, the next step is to transplant it to the real OpenFlow network environment, and more validation experiments will be done to improve the performance of the algorithm.

\section{References}

[1] GHEMAWAT S, GOBIOFF H, LEUNG S. The Google file system. ACM SIGOPS Operating Systems Review, 2003, 37(5): $29-43$.

[2] SHVACHKO K, KUANG H, RADIA S, et al. The Hadoop distributed file system. Proc. of the 26th Symposium on Mass Storage Systems and Technologies, 2010: 1-10.

[3] HAZARIKA B, SING T. Survey paper on cloud computing \& cloud monitoring: basics. SSRG International Journal of Computer Science and Engineering, 2015, 2(1): 10-15.

[4] KUDTARKAR A, SONKUSARE R, AMBAWADE D. A spindle graph based singular multipath routing using labelle merging algorithm. Proc. of the International Conference on Control, Instrumentation, Communication and Computational Technologies, 2014: 234-239.

[5] SU J, DAI B, LIU Y, et al. Inter-domain multipath routing protocols. Journal of Software, 2012, 23(1): 65-81. (in Chinese)

[6] ZHANG H, GUO X, YAN J, et al. SDN-based ECMP algorithm for data center networks. Proc. of the IEEE Computing, Communications and IT Applications Conference, 2014: $13-$ 18.

[7] CHIESA M, KINDLER G, SCHAPIRA M. Traffic engineering with equal-cost-multipath: an algorithmic perspective. Proc. of the Conference on Computer Communications, 2014: $1590-1598$.

[8] FEAMSTER N, REXFORD J, ZEGURA E. The road to SDN: an intellectual history of programmable networks. ACM Sigcomm Computer Communication Review, 2014, 44(2): $87-$ 98.

[9] DIXIT A, HAO F, MUKHERJEE S, et al. Towards an elastic distributed SDN controller. ACM Sigcomm Computer Communication Review, 2013, 43(4): 7-12.

[10] CUI W, YU Y, QIAN C. DiFS: distributed flow scheduling for adaptive switching in fat-tree data center networks. Computer Networks, 2016, 105(6): 166-179.

[11] KANAGEVLU R, AUNG K. SDN controlled local re-routing to reduce congestion in cloud data center. Proc. of the International Conference on Cloud Computing Research and Innovation, 2015: 80-88.

[12] YANG Y, YANG J, QIN D. Multipath routing algorithm for data center networks. Journal of Tsinghua University (Science and Technology), 2016, 56(3): 262-268. (in Chinese)

[13] WEI K. Research of ensemble classification methods for classimbalance and cost-sensitive datasets. Changchun, China: Jilin University, 2015. (in Chinese)

[14] GREENBERG A, HAMILTON J, JAIN N, et al. VL2: a scalable and flexible data center network. ACM Sigcomm Computer Communication Review, 2009, 39(4): 51-62.

[15] AL-FARES M, RADHAKRISHNAN S, RAGHAVAN B, et al. Hedera: dynamic flow scheduling for data center networks. Proc. of the 7th USENIX Conference on Networked Systems Design and Implementation, 2010: 1-15.

[16] WANG W, SUN Y, SALAMATIAN K, et al. Adaptive path isolation for elephant and mice flows by exploiting path diversity in datacenters. IEEE Trans. on Network and Service Management, 2016, 13(1): 5-18.

[17] LIN N, SHAO Z. Research of QoS multipath routing based on ACO algorithm. Journal of Shenyang Aerospace University,
2011, 28(6): 67-71. (in Chinese)

[18] PAKZAD F, PORTMANN M, TAN W, et al. Efficient topology discovery in OpenFlow-based software defined networks. Computer Communications, 2015, 77(1): 52-61.

[19] CHEN M, HU H, LIU B, et al. An openflow based multipath transmission mechanism. Journal of Electronics \& Information Technology, 2016, 28(5): 1242 - 1248. (in Chinese)

[20] SUBRAMANIAN R, MANORANJITHAM T. Dynamic scheduling for traffic management and load balancing using SDN. International Journal of Computer Technology and Applications, 2016, 9(2): 919-925.

[21] LI Y, PAN D. OpenFlow based load balancing for fat-tree networks with multipath support. Proc. of the IEEE International Conference on Communications, 2013: 1-5.

[22] LONG H, SHEN Y, GUO M, et al. LABERIO: dynamic loadbalanced routing in OpenFlow-enabled networks. Proc. of the 27th IEEE International Conference on Advanced Information Networking and Applications, 2013: 290-297.

[23] BENSON T, ANAND A, AKELLA A, et al. Understanding data center traffic characteristics. ACM Sigcomm Computer Communication Review, 2009, 40(1): 65-72.

[24] ZHANG C, CUI Y, TANG H, et al. State-of-the-Art survey on software defined networking (SDN). Journal of Software, 2015, 26(1): 62 -81. (in Chinese)

[25] NOSKOV A, NIKITINSKIY M, ALEKSEEV I. Development of active external network topology module for floodlight SDN controller. Modelirovanie i Analiz Informacionnyh Sistem, 2015, 22(6): $852-861$.

[26] MEDVED J, VARGA R, TKACIK A, et al. OpenDaylight: towards a model-driven SDN controller architecture. Proc. of the 15th International Symposium on a World of Wireless, Mobile and Multimedia Networks, 2014: 1-6.

[27] KAUR S, SINGH J, GHUMMAN N. Network programmability using POX controller. Proc. of the International Conference on Communiction, Computing and Systems, 2014: 134-138.

[28] ZHUANG H, DU Q. A method of data center network dynamic traffic scheduling based SDN. Journal of Computer of Modernization, 2016, (7): 80-86. (in Chinese)

[29] BHOLEBAWA I, JHA R, DALAL U. Performance analysis of proposed OpenFlow-based network architecture using mininet. Wireless Personal Communications, 2016, 86(2): $1-$ 16.

[30] PFAFF B, PETTIT J, KOPONEN T, et al. The design and implementation of openvSwitch. Proc. of the 12th USENIX Symposium on Networked Systems Design and Implementation, 2015: $116-130$

\section{Biographies}

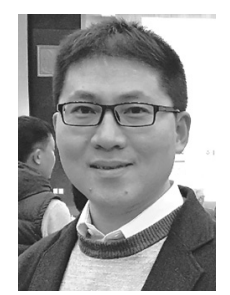

XU Xiaolong was born in 1977. He received his B.S. degree in computer and its applications, M.S. degree in computer software and theories and $\mathrm{Ph} . \mathrm{D}$. degree in communications and information systems from Nanjing University of Posts \& Telecommunications, Nanjing, China, in 1999, 2002 and 2008, respectively. He worked as a postdoctoral researcher at Station of Electronic Science and Technology, Nanjing University of Posts \& Telecommunications from 2011 to 2013. He is currently a professor in College of Computer, Nanjing University of Posts \& Telecommunications. He is a senior member of China Computer Federation. His current research interests include cloud computing and big data, mobile computing, intelligent agent and information security.

E-mail: xuxl@njupt.edu.cn 


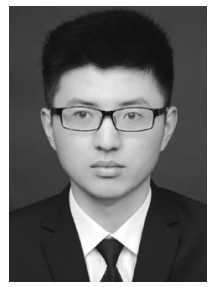

CHEN Yun was born in 1990. He received his B.E. degree in communication engineering from Jiangsu University of Science and Technology, Zhenjiang, China, in 2015. He works as an engineer in Institute of Big Data Research at Yancheng, China, carrying out research in cloud computing and software defined networking.

E-mail: 1215043122@njupt.edu.cn

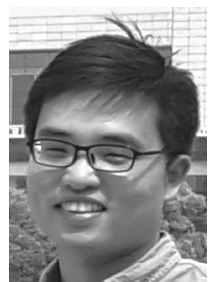

HU Liuyun was born in 1992. He received his B.E. degree in software engineering from Nanjing University of Posts \& Telecommunications, Nanjing, China, in 2016. He is now a graduate student at School of Computer Science, Nanjing University of Posts \& Telecommunications, carrying out research in cloud computing and software defined networking.

E-mail: 1216043127@njupt.edu.cn

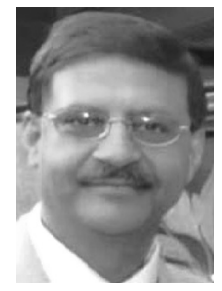

KUMAR Anup was born in 1963. He received his Ph.D. degree in Electrical and Computer Engineering Department, North Carolina State University, Raleigh, NC, in 2006. He is currently a professor and the director of Mobile Information Network and Distributed Systems Lab, Computer Engineering and Computer Science Department, University of Louisville, Louisville, USA. His current research interests include cloud computing and big data.

E-mail: anup.kumar@louisville.edu 